Proceedings of the 2011 Winter Simulation Conference

S. Jain, R.R. Creasey, J. Himmelspach, K.P. White, and M. Fu, eds.

\title{
USING ABMS TO SIMULATE EMERGENCY DEPARTMENTS
}

\author{
Paula Escudero-Marin \\ Michael Pidd \\ Department of Management Science \\ Lancaster University Management School \\ Lancaster LA1 4YX, UK
}

\begin{abstract}
Computer simulation methods have enjoyed widespread use in healthcare system investigation and improvement. Most reported applications use discrete event simulation, though there are also many reports of the use of system dynamics. There are few reports of the use of agent-based simulations (ABS). This is curious, because healthcare systems are based on human interactions and the ability of ABS to represent human intention and interaction makes it an appealing approach. Tools exist to support both conceptual modelling and model implementation in ABS and these are illustrated with a simple example from an emergency department.
\end{abstract}

\section{HEALTHCARE SYSTEM SIMULATION MODELLING}

Simulation methods have long been used to model elements of healthcare systems with a view to understanding and improvement. Literature reviews of simulation approaches in healthcare systems improvement include Fone (2003), Jun et al (1999) and Günal and Pidd (2005); and Brailsford (2007) discusses some of the challenges to be faced by those attempting such work. Brailsford follows Koelling and Schwand (2005) in dividing healthcare simulation applications into those concerned with the strategy and policy level, the tactical and operational level, and with disease prevention and epidemiology. Here we are concerned with the first two levels, which we capture under the general idea of healthcare system simulation.

\subsection{Modes of model use}

Becker et al (2005) argues that there are two intended uses of simulation models; descriptive models that are used for description, explanation and prediction; and normative models that are used for decision support purposes. However, this binary view seems too restrictive. Discussing OR/MS modelling in general, Pidd (2009) suggests four archetypal uses: decision automation, routine decision support, system investigation and improvement and providing insights for debate. Heath et al. (2009), focusing on agentbased simulation, suggests three different archetypal approaches to simulation modelling, based on the modeller's level of understanding of the system to be simulated. As shown in Figure 1, Heath et al argue that, when the level of understanding is high, a simulation can be used as a predictor; that is, as a machine that produces clear predictions about the system's behaviour under defined conditions. When the level of understanding is low, Heath et al suggests the use of a simulation model as a generator to support the generation of hypotheses and theories about system behaviour, but not in a precise manner. When the level of understanding is neither high nor low, Heath et al suggests that a simulation model be used as a mediator, which provides insight into the behaviour of the system without offering a complete representation of that behaviour. Clearly, these are three archetypal positions and many models will display characteristics that make them a mixture of predictor and mediator, or mediator and generator. These three tally, more or less, with the last three of the four types suggested by Pidd. 


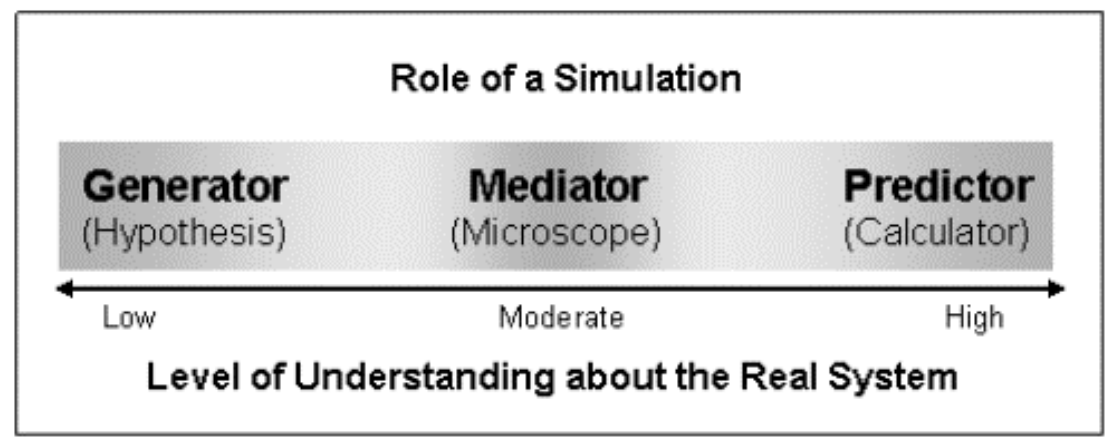

Figure 1: Roles of simulation models (from Heath et al 2009)

\subsection{Simulation modelling approaches}

Discrete event simulation (DES), system dynamics (SD) and agent-based simulation (ABS) are the main three approaches used when simulating healthcare systems. The typology of Heath et al, suggests that discrete event methods are best suited to use as predictors, or mediators, or somewhere between the two. Hence it should be no surprise that discrete event methods have enjoyed widespread use in healthcare modelling as documented in the literature reviews. Many SD models have also been developed (Brailsford 2007), though these seem to be fewer than DES models. SD models seem best suited to be used as generators; see Lane et al (2000) as an example of such an approach. What then of agent-based methods? Their use will be more formally considered later, but they seem best suited as mediators or, possibly, as generators. ABS models may offer ways to provide insight and to generate hypotheses about system behaviour by representing this as a result of the interaction of individual agents. However, the literature contains very few examples of ABS for healthcare system modelling, whatever the intended mode of use.

\section{HUMAN PERFORMANCE MODELLING}

In non-healthcare domains such as manufacturing, simulation methods have been used in human performance modelling (Kuljis, Paul, and Stergioulas 2007). Baines et al (2004) argues that errors in predicting performance in manufacturing system simulations are mainly due to inadequate representations of human behaviour in simulation models. Specifically, they suggest that it may be necessary for models to incorporate relationships between the performance of a person and the factors that affect that performance. In healthcare systems, perhaps even more than in manufacturing systems, the need for human discretion, contact and interaction suggest the importance of considering human behaviour. According to Kuljis, Paul, and Stergioulas (2007), performance metrics are taken seriously in healthcare as a way to monitor performance levels and quality of care. However, a single-dimensional focus on particular metrics, such as those related to waiting times for treatment of admission to hospital have often over-ridden reasonable clinical priorities. It seems that the performance measurement systems in place act as incentives to encourage particular aspects of performance. For example, from the mid-2000s to 2010, EDs in the UK NHS were set targets to complete the treatment of $98 \%$ of patients within 4 hours and some argued that this led to the distortion of clinical priorities. This suggests that the effect of incentives and other aspects of human performance are very important in healthcare. Therefore, in order to better understand the performance, it seems sensible to consider the inclusion of human performance factors in simulation models.

\subsection{Human performance modelling in discrete event simulation}

Some elements of human performance have long been included in DES models, usually by representing people as resources or as entities with multiple attributes that carry parameter values. The parameter 


\section{Escudero and Pidd}

values both define and are a result of human behaviour. Garnett and Bedford (2004) suggests that there are four main elements of human behaviour incorporated in DES models, albeit with limitations.

1. Human workload capacity, multi-tasking and attention span: usually focusing on the structure of a task, rather than on the person performing it.

2. Physiological and environmental factors such as age, time of the day, light, temperature or noise, which are usually outside a person's control.

3. Decision making processes: incorporated in different ways, ranging from simple 'either/or' rules to rules that allow evaluation of different strategies by linking the DES model with an expert system.

4. Human psychological factors such as motivation: including emotion, cognition and social status.

Within the healthcare system simulation literature, Brailsford and Schmidt (2003) describes a model that captures patient's motivation to attend for screening for diabetic retinopathy when offered an appointment. The model was based on a previous DES study of screening for diabetic retinopathy in a group of patients, in which the probability of attendance was constant for every patient (Davies et al. 2000). The motivation of patients to attend was implemented in the later model by adding more fields to the attributes of patient records to cover their physical, emotional, cognitive and social states. This example shows the potential for including human behaviour in DES models and leads to a more accurate way of modelling attendances for screening. However, this approach to modelling patient behaviour does not consider interactions with clinicians and others.

Another example is Günal and Pidd (2006), which describes a DES model of the process flow of patients through an A\&E department. The model represents the multitasking behaviour of medical staff by fragmenting each doctor and nurse into $\mathrm{M}$ parts so that the workload triggers multitasking behaviour. Although this is a good example of representing human behaviour and decision making processes in a model, one of the limitations of this method is that it ignores any other possible factors that may affect the multitasking behaviour. These factors may depend, for example, on the doctors' interactions with patients, other medical staff and their working environment.

\subsection{Human performance modelling in system dynamics}

Though system dynamics (SD) applications in healthcare are not as common as DES (Brailsford and Harper 2008), there is a considerable literature in this area (Bayer et al. 2007; De Vries and Beekman 1998; Gonzales-Busto and García 1999; Lattimer et al. 2004; Leischow and Milstein 2006; Sardiwal 2007). Several studies investigate the relationship between healthcare demand, healthcare capacity and waiting times under different scenarios and incorporate behavioural assumptions (Brailsford et al. 2004; Lane et al. 2000; Royston et al. 1999; Van Ackere and Smith 1999; Wolstenholme 1993, 1999).

In 1995, the OR Group of the UK Department of Health (Royston et al. 1999) began to use system dynamics to understand and improve the performance of Emergency Departments. The initial aim was to expose the structure of the UK healthcare system by considering the interactions among its different parts. The idea was to help different healthcare stakeholders develop their understanding of system behaviour resulting from system structure. This work demonstrated important relationships among different sectors of the healthcare system, such as the observation that longer waiting times in GP consultations tend to elevate the number of arrivals at emergency departments. Another important finding in this model is that behaviour changes (e.g. willingness to admit) have a greater effect than changes in capacity.

Lane et al (2000) describes the use of SD to understand the different factors contributing to the long delays for unplanned, urgent admission to acute hospitals in the UK and to explore the dynamics of the system of which the emergency department is one element. The model considers interactions between the demand pattern, ED resource deployment, other hospital processes and bed capacity. The main finding is that although some delays in the patient's pathway are inevitable, a selected augmentation of A\&E resources may lead to some reductions in those delays. Additionally, the study demonstrated that 


\section{Escudero and Pidd}

reductions in bed capacity have more impact on elective surgery cancelations than on waiting times for emergency admissions.

In summary, most SD applications have investigated the relationships between healthcare demand, healthcare capacity and within-hospital delays; others have investigated the interactions between different units of a hospital and other sectors of the healthcare system

\section{AGENT BASED MODELLING AND SIMULATION}

The term "agent" is used in different ways in different disciplines such as artificial intelligence, social science, complex science, game theory; however, there are several views about what an agent is (Borshcev and Filippov 2004). In general terms, an agent can be defined as an autonomous entity which makes decisions based on a set of rules (Bonabeau 2002). A broader definition considers an agent as an entity that possesses skills and resources and is capable of acting, perceiving and communicating, with behaviour driven by a set of tendencies (Ferber 1998). Although there are several different definitions of agent, there is some agreement: in complex adaptive systems, agents are the decision making components. That is, an agent can be seen as anything that makes choices in a system (North and Macal 2007). Moving beyond this general concept to consider agent-based modelling, an agent must be responsive, proactive and social (Wooldridge 2002). Thus agents must have attributes to make them uniquely identifiable individuals with behaviours that interact with one another to produce system behaviour. Additional characteristics such as adaptation, goal-direction and heterogeneity are often useful but not always required (Macal and North 2010).

In general, agent-based modelling and simulation (ABMS) is used to study complex systems that include agent entities whose dynamic behaviour is caused by and causes the state of the system (Shi and Brooks 2010). Although the term complexity may have different definitions, in general a complex system consists of interconnected components that work together in order to meet an objective and interchange resources and information with the environment (Tan, Wen, and Awad 2005). Therefore, in agent-based terms, a system is modelled as a set of heterogeneous agents that create overall behaviour through their interactions (Bonabeau 2002; Macal and North 2008; North and Macal 2007). An agent-based simulation is a multi-agent system in which agent behaviours and interactions are simulated for a particular purpose (Van Dyke Parunak et al. 1998; Borshcev and Filippov 2004; Macal and North 2010).

\subsection{ABMS tools}

There are several software toolkits for building ABMS applications, including free implementations of very simple approaches such as NetLogo. Published surveys of toolkits available for ABMS modelling consider aspects such as the domain where the toolkit is intended to be used, the programming language required to build the model, the possibility for visual programming and whether the software is open source (Castle and Crooks 2006; Nikolai and Madey 2009; North et al. 2006; North and Macal 2007; Serenko and Detlor 2002; Shi 2008). The Repast System (North and Macal 2007) is a widely used, open source ABMS toolkit that supports the use of several programming languages. The electronic database of literature Web of Science shows a total of 110 records for the topic "repast". Repast toolkits include tutorials to support novice users and user support networks such as mailing lists, listservs, online forums and FAQs, a library of example models, a selection of references/publications on the toolkit (Nikolai and Madey 2009). Repast Simphony (also known as Repast S) was chosen for the ABMS work in this project, though that is the subject of another paper.

\section{ABMS MODELLING OF EMERGENCY DEPARTMENTS}

A large and growing body of literature describes the use of DES and SD models in Emergency Department (ED) studies. However there are few reports of the use of ABMS for this purpose. Kanagarajah et al.(2006) describes a hypothetical ABMS of an Emergency Department intended to demonstrate the effects of fluctuations in workload and economic forces on patient safety. The agents 
included are: patients, doctors, nurses, technicians, treatment rooms and managers, and all are programmed to aim for minimizing preventable adverse events while managing patient's outcomes. Patients are attended based on their condition, the time spent by medical staff with patients depends on demand pressures and patient characteristics, and doctors may work faster or in busy periods to clear excessive queues. The interactions among the agents within the model are defined by elementary rules of decision making, movement and action.

This is a clear illustration of the potential use of ABMS in modelling EDs. As in the non ABMS model of Günal (2008), medical staff modify their behaviour based on the workload. The behaviour of the doctors is represented by changes in the speed of the work but overlooks other aspects of doctor behaviour: such as the actions or decisions that staff can take. For example, a doctor might decide to ask for more investigations on a patient in order to give a more accurate diagnosis when not under pressure to discharge a patient soon and may not do so when very busy.

Stainsby et al.(2009) describes a preliminary conceptual ABMS model of an ED model with 5 classes of agent: patients, companions of patients, administrative staff, nurses and doctors.. The conceptual model shows patient flows and interactions among agents. Stainsby et al discuss the importance of modelling human factor in emergency departments based on the idea that humans are physiologically and psychologically complex and therefore interactions among people can get much more complex. The purpose of this model is to help understand some important questions about people's behaviour in an emergency department. For instance, they suggest that using the ABMS will support increased understanding of why some patients leave an emergency department while waiting for triage, or how the implementation of a fast track system affects the level of service as perceived by the patient. The general layout of the model shows agent interaction in each stage of the pathway. The rules of interactions between agents are based on the agent that starts the interaction; though it is not clear how these interactions may occur.

\subsection{Issues to be faced in using ABMS to model emergency departments}

One of the main challenges when developing simulation models is to keep a model as simple as possible whilst including the essential of the system needed to achieve the objectives of the simulation (Robinson 2004). A further complication is that different people may have different levels of understanding of a particular system, therefore designing a model that can be accepted by different people and that allows variations in levels of complexity is a real challenge for modellers (Onggo 2010). Although there is no single accepted definition of conceptual modelling, it can be said that conceptual modelling deals with abstracting appropriate levels of simplification of a system (Robinson 2004; Pidd 1994).

Onggo (2010) classifies conceptual models into three categories: textual representations, pictorial representations and multifaceted representations. Within DES, common pictorial representations include Activity Cycle Diagrams, Process Flow Diagrams and Event Relationship Diagrams. Within SD, Causal Loop Diagrams or Stock and Flow diagrams (Sterman 2000). There exists no single pictorial scheme that provides a complete conceptual representation of an Agent Based Model (Onggo 2010). Multifaceted representations contain both diagrams and a textual representation of different conceptual model components. One of the most common multifaceted representations used in software engineering is Unified Modelling Language (UML).

\subsection{Conceptual modelling: agents and agent behaviour}

When developing ABMS it is crucial to represent the two main parts of an agent-based model: agents and agent behaviours (North and Macal 2007). Gilbert and Terna (2000) suggests that the modeller should first define the capabilities of the agents, the actions they can perform and the characteristics of the environment that surrounds them. They introduce a general scheme to build ABMS: ERA, the Environment-Rules-Agents scheme, in which the environment represents the context through rules, 


\section{Escudero and Pidd}

general data and the agents. Agent behaviour is defined by two types of rules: master rules that represent the cognition of the agent called, and maker rules that modify the master rules.

The ERA scheme can be applied to represent a general ABMS of an ED in which the main actors may be patients, nurses, doctors and managers, each with basic tasks to perform. Patients arrive at the department and can stay or leave, most likely based on their own preferences or conditions. Those who decide to stay may need to wait at any point of the process if the for their service are not yet correct. Patients also engage in diverse activities, some of which involve other patients and many of which involve clinicians and department resources. Nurses triage patients and that triage depends on the condition of the patients, and may need to be present during treatment, assessment or reassessment. As with doctors, nurses may need to multi-task, seeing multiple patients at the same time. Doctors assess, treat and reassess patients, if necessary and these tasks may depend on factors such as: patient condition, time of the day, patient's time in the system, internal regulations and standards imposed by the department and personal attitudes. Therefore when doctor is choosing a patient to asses may consider not only the clinical priorities of patients but these other factors too. As with nurses, doctors may switch between patients to see multiple patients simultaneously.

\subsection{Conceptual modelling: ABMS implementation}

The context contains the different agents which interact to each other using the environment: for example Patients, Nurses, Doctors and the Department (which plays the role of manager). Each agent has simple rules of behaviour. For instance, a particular doctor may choose a patient based on a set of specific master rules based on the condition of the patient and the time spent by that patient within the department. However, that doctor can adapt or learn from the system and modify those rules using a set of maker rules. Figure 2 shows a simple ERA scheme for an Emergency Department model.

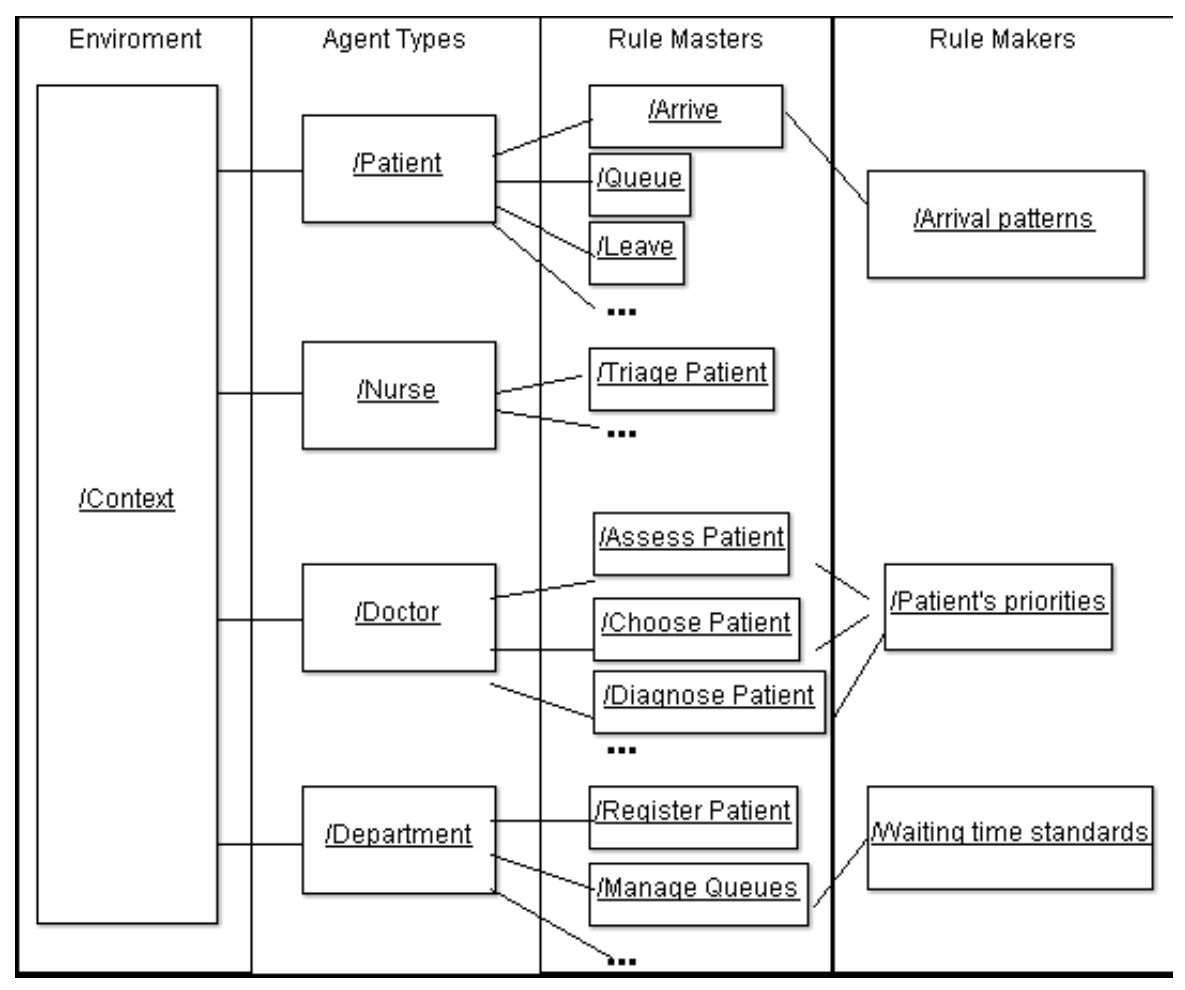

Figure 2: ERA scheme for an Emergency Department 


\section{Escudero and Pidd}

Once the agents and their behaviour are defined, it is necessary to develop a model detailed conceptual representation that can form the basis for model coding. ABMS are usually implemented in an object-oriented platform, for which UML is a generally accepted form of conceptual representation. (Bauer and Odell 2005). An advantages of using UML in modelling is that its symbols facilitate the verification and validation process, moreover the use of an internationally standardized language can elicit common understanding among different stakeholders (Martin et al. 2011).

UML 2.0 includes thirteen types of diagrams to represent static application structure, behaviour and interactions (Onggo 2010) of which Use Case Diagrams, State Diagrams, Activity Diagrams, Class Diagrams, and Object Diagrams appears useful in ABMS (North and Macal 2007). Onggo (2010) suggests, in addition, that Sequence Diagrams and Collaboration Diagrams can be also used to represent interactions of the model.

Use Case Diagrams provide a specification of actions that agents can perform with outside actors (Odell, Van Dyke Parunak, and Bauer 2001) as shown in Figure 3.

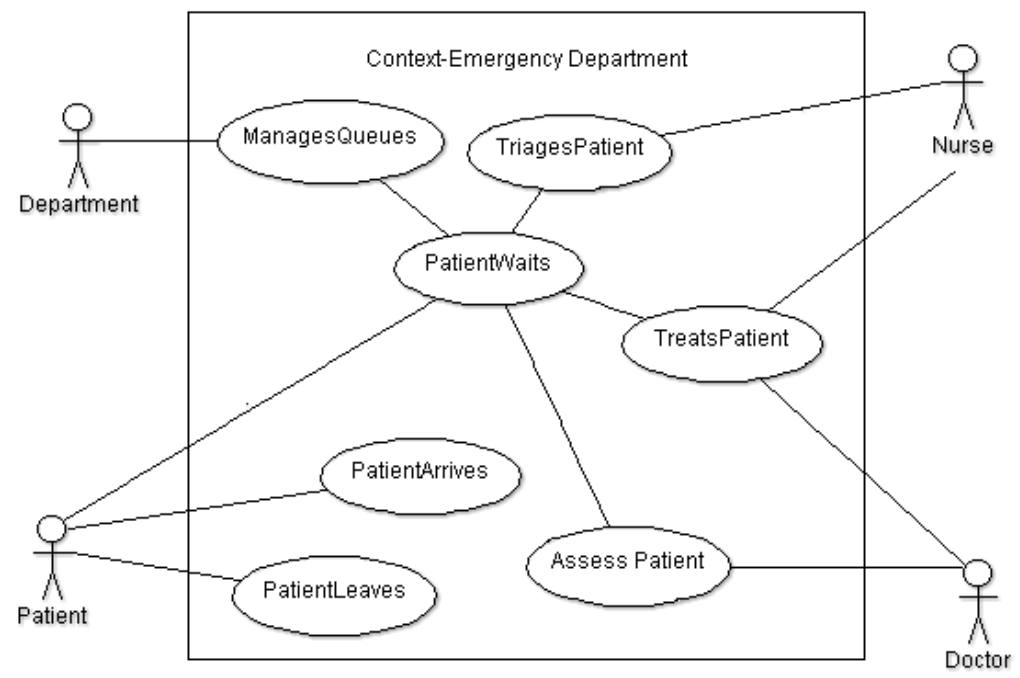

Figure 3: Example of a Use Case Diagram for an Emergency Department

A Class Diagram can be used to specify the available properties and potential behaviours of agents. This static structure, shows inheritance sources or super-classes. In this example there is a single superclass called GenericAgent that possesses general characteristics and methods that are inherited by the subclasses: PatientAgent, Doctor, Nurse and Department. Those sub-classes also have their own properties and methods (See Figure 4). 


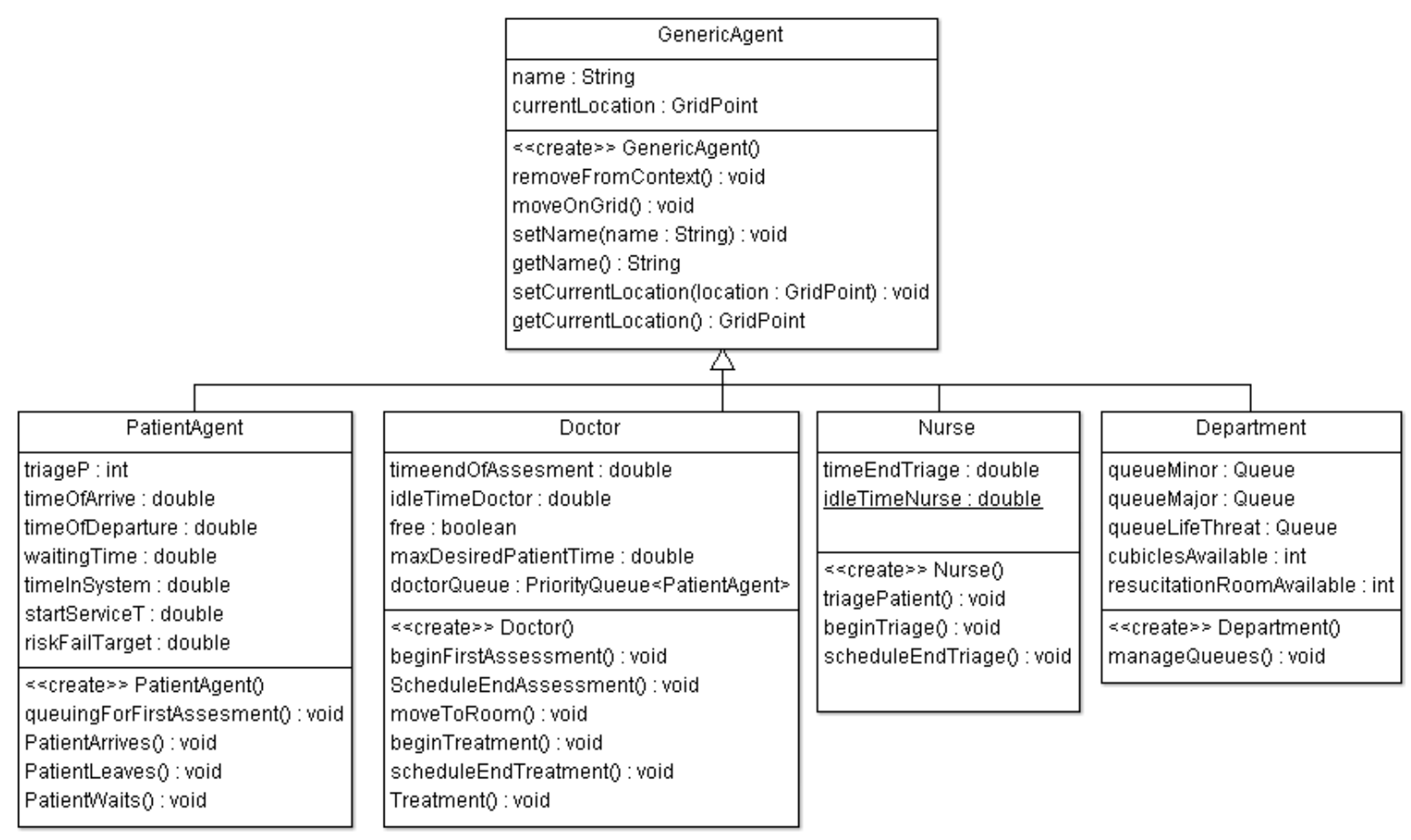

Figure 4: Example of a Class Diagram for an Emergency Department

\section{CONCLUSIONS}

It seems that there is considerable scope for the use of agent-based simulation approaches in healthcare system modelling, though there are very few reports of its use. Such models sit between the detail that is possible in a discrete event simulation and the broader treatment of system dynamics models. This paper has provided a general description of the possible potential of ABMS in healthcare, linking this to different modes of model use. Given the importance of human interaction and intention in emergency care, there is an opportunity for people skilled in agent-based modelling and simulation to make a contribution towards its improvement.

As with all approaches to computer simulation, before descending into model coding, it seems important to dwell on the conceptualisation of a model, though there is, as yet, no widely agreed approach to this for ABMS. As shown here, UML-based multi-facetted representations have much to commend them, given the somewhat complex interactions and behaviours to be represented in an ABS model and the need to link agent classes to their behaviours, whilst maintaining a conceptual distinction between the agents and their behaviours..

AMBS software support is continually improving, ranging from simple systems such as NetLogo and fully configured toolkits such as Repast Simphony. The latter includes some tools to support graphical model building, but these are nowhere near the level of sophistication and ease of use enjoyed by developers of discrete event and system dynamics simulations.

This suggests that more widespread use of agent-based approaches in healthcare system simulation will depend on the development of better and more widely adopted conceptualisation and implementation tools. It will also depend on the availability of trained modellers, though it seems likely that this will in turn depend, in part at least, on developments in conceptualisation and implementation. 


\section{REFERENCES}

Baines, T, S Mason, PO Siebers, and J Ladbrook. 2004. "Humans: the missing link in manufacturing simulation?" Simulation Modelling Practice and Theory no. 12 (7-8):515-526.

Bauer, B., and J. Odell. 2005. "UML 2.0 and agents: how to build agent-based systems with the new UML standard." Engineering applications of artificial intelligence no. 18 (2):141-157.

Bayer, S., M. Köberle-Gaiser, and J. Barlow. 2007. "Planning for Adaptability in Healthcare Infrastructure." Paper presented at The 25th International System Dynamics Conference, System Dynamics Society, at Boston, USA.

Becker, J., B. Niehaves, and K. Klose. 2005. "A framework for epistemological perspectives on simulation." Journal of Artificial Societies and Social Simulation no. 8 (4):1.

Bonabeau, E. 2002. "Agent-based modeling: Methods and techniques for simulating human systems." Proceedings of the National Academy of Sciences of the United States of America no. 99 (Suppl 3):7280.

Borshcev, A. , and A. Filippov. 2004. "From System Dynamics and Discrete Event to Practical Agent Based Modeling: Reason, Techniques, Tools." Paper presented at The 22nd International Conference of the System Dynamics Society, July 25 - 29, 2004, at Oxford, England.

Brailsford, S, and P Harper. 2008. "OR in Health." European Journal of Operational Research no. 185 (3):901-904.

Brailsford, S, and B Schmidt. 2003. "Towards incorporating human behaviour in models of health care systems: an approach using discrete event simulation." European Journal of Operational Research no. 150 (1):19-31.

Brailsford, S. 2007. "Tutorial: Advances and Challenges in Healthcare Simulation Modelling." In Proceedings of the 2007 Winter Simulation Conference, edited by Scott J. Mason, Raymond R. Hill, Lars Mönch, Oliver Rose, Thomas Jefferson and John W. Fowler, 1436-1448. Piscataway, New Jersey: Institute of Electrical and Electronics Engineers.

Brailsford, S., V. Lattimer, P. Tarnaras, and C. Turnbull. 2004. "Emergency and On-Demand Health Care: Modelling a Large Complex System." Journal of the Operational Research Society no. 55 (1):34-42.

Castle, C. and A. Crooks. 2006. "Principles and concepts of agent-based modelling for developing geospatial simulations." Centre for Advanced Spatial Analysis, University College London, September 2006.

Davies, R, S Brailsford, P Roderick, C Canning, and D Crabbe. 2000. "Using simulation modelling for evaluating screening services for diabetic retinopathy." Journal of the Operational Research Society no. 51 (4):476-484.

De Vries, T., and R. Beekman. 1998. "Applying simple dynamic modelling for decision support in planning regional health care." European Journal of Operational Research no. 105:277-284.

Ferber, J. 1998. Multi-agent systems: an introduction to distributed artificial intelligence: AddisonWesley.

Fone, D, S Hollinghurst, M Temple, A Round, N Lester, A Weightman, K Roberts, E Coyle, G Bevan, and S Palmer. 2003. "Systematic review of the use and value of computer simulation modelling in population health and health care delivery." Journal of Public Health, 25 (4):325.

Garnett, J., and T. Bedford. 2004. "Towards a hybrid modelling system of human performance." Paper presented at the UK Operational Research Society Simulation Workshop (SW2004), at Hornton Grange, Birmingham, UK. 23-24th March 2004.

Gilbert, N, and P Terna. 2000. "How to build and use agent-based models in social science." Mind \& Society no. 1 (1):57-72.

Gonzales-Busto, B., and R. García. 1999. "Waitin Lists in Spanish Public Hospitals: A System Dinamics Approach." System Dynamics Review no. 15 (3):201-224.

Günal, M. 2008. Simulation Modelling for Understanding Performance in Healthcare, Department of Management Science, Lancaster University, Lancaster. 
Günal, M., and M. Pidd. 2006. "Understanding Accident and Emergency Department Performance Using Simulation." In Proceedings of the 2006 Winter Simulation Conference, at Monterey, CA, edited by David Nicol, Richard Fujimoto, Barry Lawson, Jason Liu, Felipe Perrone and Fred Wieland, 446-452. Piscataway, New Jersey: Institute of Electrical and Electronics Engineers.

Günal, M.M., and M. Pidd. 2005. "Simulation modelling for performance measurement in healthcare." In Proceedings of the 2005 Winter Simulation Conference, edited by M. E. Kuhl, N. M. Steiger, F. B. Armstrong, and J. A. Joines, 2663-2668. Piscataway, New Jersey: Institute of Electrical and Electronics Engineers, Inc.

Heath, B., R. Hill, and F. Ciarallo. 2009. "A survey of agent-based modeling practices (january 1998 to july 2008)." Journal of Artificial Societies and Social Simulation no. 12 (4):9.

Jun, JB, SH Jacobson, and JR Swisher. 1999. "Application of discrete-event simulation in health care clinics: A survey." Journal of the Operational Research Society:109-123.

Kanagarajah, AK, PA Lindsay, AM Miller, and DW Parker. 2006. "An exploration into the uses of agentbased modeling to improve quality of health care." In Proceedings of the 6th International Conference on Complex Systems, at Boston, MA, Edited by A. Minai, D. Braha and Y. Bar-Yam, 10 p. The New England Complex Systems Institute.

Koelling, P., and M. Schwandt. 2005. "Health Systems: A Dynamic System - Benefits from System Dynamics." In Proceedings of the 2005 Winter Simulation Conference, at Orlando, Fl, edited by M. E. Kuhl, N. M. Steiger, F. B. Armstrong, and J. A. Joines, 1321-1327. Piscataway, New Jersey: Institute of Electrical and Electronics Engineers, Inc.

Kuljis, J, RJ Paul, and LK Stergioulas. 2007. "Can health care benefit from modeling and simulation methods in the same way as business and manufacturing has?" In Proceedings of the 2007 Winter Simulation Conference, at Washington, D.C., edited by S. G. Henderson, B. Biller, M.-H. Hsieh, J. Shortle, J. D. Tew and R. R. Barton, 1449-1453. Piscataway, New Jersey: Institute of Electrical and Electronics Engineers, Inc.

Lane, D., J. Monefeldt, and V. Rosenhead. 2000. "Looking in the Wrong Place for Healthcare Improvements: A System Dynamics Study of an Accident and Emergency Department." Journal of the Operational Research Society no. 51 (5):518-531.

Lattimer, V., S. Brailsford, J. Turnbull, P. Tarnaras, H. Smith, S. George, K. Gerard, and S. MashlinProthero. 2004. "Reviewing emergency care systems I: insigths from system dynamics modelling." Emergency Medicine Journal, 21:685-691.

Leischow, S., and B. Milstein. 2006. "Systems Thinking and Modeling for Public Health Practice." American Journal of Public Health no. 96 (3):403-405.

Macal, CM, and MJ North. 2008. "Agent-based modeling and simulation: ABMS examples." In Proceedings of the 2008 Winter Simulation Conference, at Miami, Miami, FL, edited by S. J. Mason, R. R. Hill, L. Mönch, O. Rose, T. Jefferson and J. W. Fowler, 101-112. Piscataway, New Jersey: Institute of Electrical and Electronics Engineers, Inc.

- 2010. "Tutorial on agent-based modelling and simulation." Journal of Simulation no. 4 (3):151162.

Martin, M., R. Champion, L. Kinsman, and K. Masman. 2011. "Mapping patient flow in a regional Australian emergency department: A model driven approach." International Emergency Nursing no. 19 (2):75-85.

Nikolai, C, and G Madey. 2009. "Tools of the trade: A survey of various agent based modeling platforms." Journal of Artificial Societies and Social Simulation no. 12 (2):2.

North, M., N. Collier, and J. Vos. 2006. "Experiences creating three implementations of the repast agent modeling toolkit." ACM Transactions on Modeling and Computer Simulation (TOMACS) no. 16 (1):1-25.

North, M., and C. Macal. 2007. Managing Business Complexity: Discovering strategic solutions with Agent-Based Modeling and Simulation. New York: Oxford University Press, Inc. 
Odell, J., H. Van Dyke Parunak, and B. Bauer. 2001. Representing agent interaction protocols in UML." In Agent-Oriented Software Engineering, edited by P. Ciancarini and M. Wooldridge, 201-218. Springer.

Onggo, S. 2010. "Methods for conceptual model representation." In Conceptual Modelling for DiscreteEvent Simulation, edited by Brooks RJ, Robinson S, Kotiadis K and van der Zee D-J and Taylor and Francis. CRC Press.

Pidd, M. 2009. Tools for thinking: modelling in management science. Third edition ed. Chippenhm, Wiltshire, UK: John Wiley \& Sons Ltd.

Pidd, Michael. 1994. "An introduction to computer simulation." In Proceedings of the 1994 Winter Simulation Conference at Orlando, Florida, United States, Edited by Deborah A. Sadowski, Andrew F. Seila, Jeffrey D. Tew and S. Manivannan, 7-14. Piscataway, New Jersey: Institute of Electrical and Electronics Engineers, Inc.

Robinson, S. 2004. Simulation: the practice of model development and use.: John Wiley \& Sons, Ltd.

Royston, Geoff., A. Dost, J. Townshend, and H. Turner. 1999. "Using System Dynamics to help develop and implement policies and programmes in health care in England." System Dynamics Review no. $15(3): 293-313$.

Sardiwal, S. 2007. "Conceptualization and formulation of a UK health and social care system using System Dynamics." In Proceedings of the the 2007 System Dynamics Conference., July 29 August 2, 2007, at Boston, Massachusetts, USA, Edited by J. Sterman. Curran Associates, Inc.

Serenko, A, and B Detlor. 2002. "Agent toolkits: A general overview of the market and an assessment of instructor satisfaction with utilizing toolkits in the classroom." In Working Paper 455. Ontario, Canada: McMaster University.

Shi, DF. 2008. Determining the range of predictions for calibrated Agent-Based Simulation Models. PhD thesis, Management Science, Lancaster University, Lancaster.

Shi, DF, and RJ Brooks. 2010. "Determining The Range Of Predictions For Calibrated Agent-Based Simulation Models." In Operational Research Society Simulation Workshop 2010, 23-24 March 2010, at Worcestershire, England, Edited by, 17-22. Operational Research Society.

Stainsby, H, M Taboada, and E Luque. 2009. "Towards an Agent-Based Simulation of Hospital Emergency Departments." Paper presented at the 2009 IEEE International Conference on Services Computing (SCC 2009), 21-25 September 2009, at Bangalore, India.

Sterman, J. 2000. Business Dynamics: Systems Thinking and Modeling for a Complex World: McGrawHill

Tan, J., H.J. Wen, and N. Awad. 2005. "Health Care and Services Delivery Systems as Complex Adaptive Systems." Communications of the ACM, 36-44.

Van Ackere, A., and P. Smith. 1999. "Towards a macro model of National Health Service waiting lists." System Dynamics Review no. 15 (3):225-252.

Van Dyke Parunak, H, R Savit, and RL Riolo. 1998. "Agent-based modeling vs. equation-based modeling: A case study and users' guide." In Proceedings of Multi-agent systems and Agentbased Simulation (MABS'98), July 4-6, 1998, at Paris, France, edited by Jaime S.; Conte Sichman, Rosaria; Gilbert, Nigel 10-25. Springer.

Wolstenholme, E. 1993. "A case Study in Community Care Using System Thinking." The Journal of the Operational Research Society no. 44 (9):925-934.

1999. "A patient flow perspective of UK Health Services: Exploring the case for new "immediate care" initiatives." System Dynamics Review no. 15 (3):253-271.

Wooldridge, M.J. 2002. An introduction to multiagent systems: Wiley. 


\section{AUTHOR BIOGRAPHIES}

MICHAEL PIDD is Professor of Management Science and Head of the Department of Management Science at Lancaster University Management School. He is known for his writings on simulation modelling, including the books Computer Simulation in Management Science and Tools for Thinking: Modelling in Management Science, both of which have been in print for many years. His current research interests include simulation modelling in healthcare, technical developments in simulation modelling methodology and performance measurement in public services. He is a past-President of the UK Operational Research Society. His email address is m.pidd@lancaster.ac.uk and his personal homepage is at $<$ http://www.lancs.ac.uk/staff/smamp $>$.

PAULA ESCUDERO-MARIN is a $\mathrm{PhD}$ student of Management Science at Lancaster University Management School. She obtained a Master in Systems Engineering and a B.S. degree in Industrial Engineering from Universidad Nacional in Colombia. She is a lecturer at Eafit University in Colombia. She is currently a recipient of a COLCIENCIAS-LASPAU scholarship to pursue her PhD studies at Lancaster University. Her research interests include Discrete-Event Simulation, System Dynamics and Agent Based Modelling and Simulation. Her email address is p.escuderomarin@lancs.ac.uk and her personal homepage is at $<$ http: //www. lums. lancs.ac.uk/profiles/paula-escudero/>. 